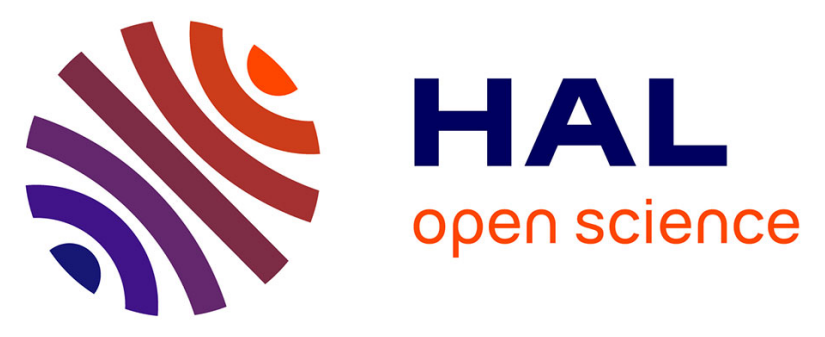

\title{
Synergistic Effect of Small Molecules on Large-Scale Structure of Simplified Industrial Nanocomposites
}

Dafne Musino, Anne-Caroline Genix, Caroline Fayolle, Aurelie Papon, Laurent Guy, Natalia Meissner, Radoslaw Kozak, Pawel Weda, Thomas Bizien, Thomas Chaussee, et al.

\section{To cite this version:}

Dafne Musino, Anne-Caroline Genix, Caroline Fayolle, Aurelie Papon, Laurent Guy, et al.. Synergistic Effect of Small Molecules on Large-Scale Structure of Simplified Industrial Nanocomposites. Macromolecules, 2017, 50 (13), pp.5138-5145. 10.1021/acs.macromol.7b00954 . hal-01580193

\section{HAL Id: hal-01580193 \\ https://hal.science/hal-01580193}

Submitted on 16 Nov 2018

HAL is a multi-disciplinary open access archive for the deposit and dissemination of scientific research documents, whether they are published or not. The documents may come from teaching and research institutions in France or abroad, or from public or private research centers.
L'archive ouverte pluridisciplinaire HAL, est destinée au dépôt et à la diffusion de documents scientifiques de niveau recherche, publiés ou non, émanant des établissements d'enseignement et de recherche français ou étrangers, des laboratoires publics ou privés. 


\section{Synergistic effect of small molecules on large-scale structure of simplified industrial nanocomposites}

Dafne Musino ${ }^{1}$, Anne-Caroline Genix ${ }^{1} *$, Caroline Fayolle ${ }^{2}$, Aurélie Papon ${ }^{2}$, Laurent Guy ${ }^{2}$, Natalia Meissner $^{3}$, Radosław Kozak ${ }^{3}$, Pawel Weda ${ }^{3}$, Thomas Bizien ${ }^{4}$, Thomas Chaussée ${ }^{2}$, Julian Oberdisse ${ }^{1}$

${ }^{1}$ Laboratoire Charles Coulomb (L2C), UMR 5221 CNRS, Université de Montpellier, F-34095

Montpellier, France

${ }^{2}$ Solvay Silica, 15 rue Pierre Pays BP52, 69660 Collonges au Mont d'Or, France

${ }^{3}$ Synthos Spótka Akcyjna, Chemików 1, 32600 Oświęcim, Poland

${ }^{4}$ SOLEIL Synchrotron, L'Orme des Merisiers, Gif-Sur-Yvette, 91192 Saint-Aubin, France

*Author for correspondence: anne-caroline.genix@umontpellier.fr 


\begin{abstract}
The microstructure of polymer nanocomposites made with disordered silica filler (Zeosil(R) 1165MP) of industrial relevance and various coating agents is quantitatively analyzed using a combination of SAXS, TEM, and a recently developed structural model. The polymer matrix is formed by an endfunctionalized styrene-butadiene statistical copolymer capable of covalent grafting on the silica nanoparticles. The effect of the coating agents with different alkyl chain length $\left(\mathrm{C}_{8}, \mathrm{C}_{12}\right.$, and $\left.\mathrm{C}_{18}\right)$ on filler structure quantified in terms of aggregate formation, for different concentrations (up to $8 \% \mathrm{wt}$ with respect to silica), and the effect of a commonly added catalyzer, DPG, are studied using the structural model. As a result we show that a strongly synergetic effect of both DPG and coating agent exist. Our findings open the road to a fundamental understanding and rational design of model and industrial nanocomposite formulation with optimized coating agents.
\end{abstract}




\section{Introduction}

The composition of modern car tire tread materials is of great complexity, and little is known about the impact of each of the many ingredients on the final structure and performance of such polymer nanocomposites (PNC) ${ }^{1}$, in spite of many fundamental studies of such materials ${ }^{2-7}$. For the industrial application, performance is usually quantified in terms of specific mechanical properties related to, e.g., rolling resistance or wet grip. ${ }^{8-10}$ Since the advent of highly dispersible silica some twenty years ago, performance of such materials has been boosted ${ }^{11-14}$. Silanol surface groups on the silica result in a strong filler-filler interaction though at the detriment of the filler-polymer interaction. ${ }^{15}{ }^{16}$ The latter is dominant, e.g., for the traditional car tire filler - carbon black -, which display a high dispersive contribution of the surface energy. To overcome this disadvantage of the silica, which is due to the mismatch in polarity between the hydrophilic nanoparticle surfaces and the hydrophobic polymer, an obvious solution was found with the addition of coating and coupling agents. The firsts are small amphiphilic silane molecules playing a role analogous to surfactant molecules in, e.g., microemulsions. They are known to decrease both dispersive and polar contributions of the surface energy, whereas the introduction of a coupling agent compensates the decrease of the dispersive part, as compared to carbon black, by providing covalent links of the filler nanoparticles to the polymer matrix. ${ }^{17}$ These agents are commonly used in modern car tires, and numerous studies have evidenced their impact on dispersion and macroscopic properties, see e.g., ${ }^{18-21}$. Note that in some cases, different surface chemistries, for instance with phosphonic acids, have been developed in order to adapt surface modification strategies to, e.g., aqueous environments ${ }^{22}$, before casting the nanocomposite samples, and its effect on the final nanocomposite structure ${ }^{23}$.

Several studies have been dedicated to the impact of (in situ) filler-surface modification with silanes on the mechanical properties of filled styrene-butadiene (SB) prepared by solid-phase mixing, sometimes reporting impact on microstructure, sometimes not. ${ }^{12,24-30}$ Such systems are close to the industrial application, usually with a crosslinked matrix (rubber, SBR), involving the addition of various additives like, e.g., zinc oxide, sulfur, CBS (cyclohexyl benzothiazole sulfenamide) and DPG (diphenyl guanidine), referred to as 'curing package' ${ }^{25}$. Stöckelhuber et al ${ }^{25}$ have studied SBR filled with different silica nanoparticles and silane grafting, and provided evidence by dynamical mechanical measurements for the impact of the interfacial properties on large-scale mechanical properties and in particular the Payne effect ${ }^{31}$. The Payne effect in silica-filled SBR was also studied as a function of surface modification with coating or coupling agents by Ramier et al ${ }^{24}$. In this case, the dispersion state of the silica was not found to vary, whereas the amplitude of the Payne effect was found to decrease with surface modification, as also observed by Gauthier et al ${ }^{28}$. The reversible decrease of elastic modulus associated with the Payne effect was correlated with bound rubber measurements and discussed in terms of chain desorption from the filler surface. 
Several groups have observed correlations of microscopic structure with mechanical properties. ${ }^{21,26,32 \text {, }}$ ${ }^{33} \mathrm{Wu}$ et al have studied the influence of surface modification of silica filler with a coupling agent in SBR. ${ }^{21}$ They have shown that an in situ modification of silica NPs with coupling agent increases the content of bound rubber, and improves the dispersion of silica, the stability of which is ensured by the chemical bonds between filler and rubber. They also provided rheological evidence for a simultaneous improvement of both rolling resistance and wet grip, i.e., the industrially relevant quantities. Conzatti et al have studied the impact of surface modification of silica in SBR on structure by TEM. ${ }^{33,34}$ They have shown that the better dispersion obtained with silane agents is correlated with a shift in percolation threshold towards higher filler contents, as well as a higher tensile strength at high deformation. Finally, the use of a coating agent to reduce filler-filler interactions and improve dispersion in SBR was exploited by Mujtaba et al. ${ }^{35}$ This induces a higher specific surface which contributes strongly to mechanical properties, whereas an additional fraction of micron-sized fillers is found to have little effect. In all these examples, the SBR nanocomposites display a complex formulation - including curing agents as mentioned above -, and the grafting of coating or coupling agents onto the silica surfaces cannot be studied independently.

On the contrary, the impact of individual curing ingredients on rheology has been investigated by Schaal et al. ${ }^{36}$ They followed rheological changes upon annealing and found that DPG leads to a retardation of the presumed re-agglomeration of silica particles. DPG is a basic molecule traditionally used as a secondary accelerator, but it was also found to improve the silanization reaction during mixing. ${ }^{37}$ This is beneficial for the curing process as higher coverage of the silica surface allows better efficiency of the accelerator (e.g., CBS), which otherwise tends to adsorb on the silica surface. ${ }^{38}$ It follows that the mixing order of the ingredients is of primary importance. If, in many cases, DPG was added together with the curing package - i.e., at the end of the mixing process ${ }^{26,27,35,39}$ or later by using a two-roll mill ${ }^{24,36,40}$-, it was recently introduced in the first stage of mixing together with the coating ${ }^{41}$ or coupling ${ }^{19}$ agent and the silica, following a patented innovation by Michelin ${ }^{37}$. To the best of our knowledge, however, no quantitative study of filler dispersion and aggregation in styrenebutadiene of the separate and combined impact of both coating agent and DPG on the final PNC structure exists. This is the objective of the present letter.

In recent contributions, we have developed a combined TEM-SAXS-simulation analysis of nanoparticle and aggregate dispersions in systems of disordered silica in styrene-butadiene copolymer of industrial relevance but with a limited number of ingredients ${ }^{41-44}$. Motivated, in particular by the absence of any additive related to curing but DPG, the system with un-crosslinked SB matrix was termed 'simplified industrial'. The objective of the present letter is to use our quantitative analysis to characterize the structure of the filler particles in such systems as a function of the concentration of small molecules, i.e., coating agent and DPG, in order to investigate their - empirically known to be synergetic - impact on nanocomposite structure. 


\section{Materials and methods}

Sample formulation. Styrene-butadiene random copolymer was purpose-synthesized by Synthos in two highly monodisperse versions (polydispersity index $=1.02$ ): one non functionalized of molecular weight $177 \mathrm{~kg} / \mathrm{mol}$ (denoted 'NF'), the other (170 kg/mol, denoted ' $\mathrm{F}$ ') with a single graftable endfunction $\mathrm{SiMe}_{2}-\mathrm{OH}$, also called D3. The synthesis protocol is outlined elsewhere. ${ }^{42}$ An industrially relevant example of a coating agent is octyltriethoxysilane ("octeo"), which is typically used at $8 \%$ wt with respect to silica, and the content of which is varied in the present study from zero to the latter value $(0,4 \%, 8 \%)$. The mass of the short aliphatic chain of the silane was also varied, from octyl- to dodecyl- and octadecyl-triethoxysilane (termed $\mathrm{C}_{8}$ from Sigma-Aldrich, $\mathrm{C}_{12}$ and $\mathrm{C}_{18}$ from Alfa Aesar, of molar mass 276, 332, and $417 \mathrm{~g} / \mathrm{mol}$, resp.), keeping the molar concentrations constant with respect to the $8 \%$ wt octeo samples. The filler is obtained by fragmentation of precipitated silica micropellets (Zeosil(R) $1165 \mathrm{MP}$ from Solvay), which have a nominal specific surface of $160 \mathrm{~m}^{2} / \mathrm{g}$. The size distribution of the primary nanoparticles obeys a log-normal law in agreement with TEM studies $\left(\mathrm{R}_{0}=\right.$ $8.55 \mathrm{~nm}, \sigma=27 \%$, leading to an average bead volume of $\left.\mathrm{V}_{\mathrm{Si}}=3.610^{3} \mathrm{~nm}^{3}\right) .{ }^{41}$ Finally, 1,3-diphenyl guanidine (DPG from Bayer, $211 \mathrm{~g} / \mathrm{mol}, 1 \% \mathrm{wt}$ with respect to polymer) was also used during the mixing process. As discussed above, its empirical role is to enhance silanization. It is the second small molecule the impact of which is investigated here, on its own and in combination with octeo to characterize the potential synergy. PNC samples were produced by stepwise introduction and mixing in an internal mixer at a temperature reaching $155^{\circ} \mathrm{C}$ to ensure silanization ${ }^{45}$. The polymer was introduced first at $70^{\circ} \mathrm{C}$ and masticated for 1 min before introduction in two equal steps of the silica together with the coating agent and DPG. Mixing is followed by two passages between rotating cylinders at $70^{\circ} \mathrm{C}$ first, then another twelve passages after cooling to room temperature. Finally, samples have been pressed at $\mathrm{T}=60^{\circ} \mathrm{C}, \mathrm{P}=60$ bar for $3 \mathrm{~min}$, to reach a final sample thickness of about $1.5 \mathrm{~mm}$. Effective grafting after the mixing process was checked by bound rubber (BR) measurements giving the quantity of non-extractable chains. The BR fraction has been determined by extraction of the free chains in xylene and measuring the resulting sample mass after evaporation of the solvent. This indicator for the efficiency of chain grafting on the silica surface is also linked to the structuration of the filler ${ }^{42}$. Note that due to the absence of antioxidant agent in the formulation, some weak crosslinking of SB occurs during mixing resulting in an incomplete dissolution of the matrix polymer in a good solvent of the polymer (THF). For comparison, some crosslinked samples have also been produced by adding sulfur and CBS (1.5\% wt with respect to polymer for each) after the internal mixing step. In order to reduce the number of parameters, a single silica volume fraction of $\Phi_{\mathrm{Si}} \approx$ $18 \% \mathrm{v}$ has been investigated here. For all samples, the volume fractions characterized by thermogravimetric analysis display some small spread of no more than $0.3 \% \mathrm{v}$.

Structural analysis. Small-angle X-ray scattering was performed on beamline SWING at synchrotron SOLEIL (Saint Aubin, France) using standard conditions (sample-to-detector distances $2 \mathrm{~m}, 5 \mathrm{~m}$ and 
$6.5 \mathrm{~m}$; wavelength $1 \AA$, giving a q-range from $6.210^{-4} \AA^{-1}$ to $5.610^{-1} \AA^{-1}$ ). Standard data reduction tools given by Soleil were used (Foxtrot 3.1). Matrix contributions have been measured independently, and subtracted. Transmission electron microscopy (TEM) was performed on samples prepared by ultracryomicrotomy at $-120^{\circ} \mathrm{C}$ (desired thickness set to $90 \mathrm{~nm}$, copper grids with carbon membrane) using a FEI Tecnai Biotwin at $120 \mathrm{kV}$. SAXS reveals a multi-scale filler structure: NPs forms aggregates which are themselves concentrated within fractal branches extending over micrometers. In the quantitative structural model that we have developed ${ }^{41}$, the central element is the aggregate defined by its internal characteristics, namely average compacity $\kappa$, and aggregation number $\mathrm{N}_{\text {agg. }}$. The SAXS analysis is performed in two steps: (i) the use of a Kratky plot ( $q^{2} I(q)$ versus $q$ ) to extract the average aggregate radius $R_{\text {agg }}$, and (ii) the description of the inter-aggregate interaction by a simulated structure factor for polydisperse spheres representing aggregates with hard sphere interactions. As the silica fraction is fixed here, the latter contribution has a minor impact, and changes in the SAXS data are mainly related to a different aggregate form factor (mass and size). Comparison with the SAXS intensity at a low-q value leads to the determination of the average aggregate compacity. ${ }^{23,41}$ Note that quantitative input of TEM in terms of the volume fraction of fractal branches $\Phi_{\text {fract }}$ (or equivalently the volume fraction of pure polymer zones, given by $1-\Phi_{\text {fract }}$ ) is needed to estimate the internal volume fraction, $\Phi_{\text {agg }}=\Phi_{\mathrm{Si}} /\left(\kappa \Phi_{\text {fract }}\right){ }^{41}$ On average for the samples with functionalized chains, $\Phi_{\text {fract }}$ about $80 \pm 2 \%$ was found. For NF and 50/50, we used $\Phi_{\text {fract }}=58$ and $73 \%$, respectively ${ }^{42}$.

\section{Results and discussion}

Impact of matrix composition on nanocomposite structure. In recent studies of simplifiedindustrial nanocomposites, we have investigated the impact of chain parameters on the filler aggregate structure by SAXS, and in particular chain end-functionalization ${ }^{42}$ and chain mass ${ }^{46}$. In order to test the influence of the coating agent and DPG reported in the present letter, a matrix composition had to be chosen. As the chains had been newly synthesized with a slightly different chain mass than before ${ }^{41,44}$, the impact of end-functionalization was studied first, in order to choose the most promising system in terms of structural evolution. For the same reason, we have chosen to work with a high volume fraction of silica filler $\left(\Phi_{\mathrm{Si}}=18 \% \mathrm{v}\right)$. In Figure 1, three normalized SAXS intensity curves are shown, corresponding to either a pure non functionalized matrix (NF), a fully functionalized one (F), and a 50/50 mixture. The structural features of these nanocomposites are clearly recognized in the scattered intensities: the high-q scattering is reminiscent of the primary silica NPs, identical in all formulations. A small bump in the range $0.02-0.03 \AA^{-1}$ (well visible in a Kratky representation, see later in Figure $3 b$ ) characterizes the interactions between particles in contact, and below that q-value aggregate scattering is dominant. A much stronger change in slope around $0.01 \AA^{-1}$ is indicative of aggregate-aggregate interactions, and this position in q-space is used to extract the average aggregate radius $R_{\text {agg }}$ from the scattering ${ }^{41}$. In this intermediate q-range, the strong influence of the matrix 
composition on the structure is visible. At low-q, finally, a low-q upturn typical for large-scale filler structures is visible.



Figure 1: Reduced SAXS intensities $\mathrm{I}(\mathrm{q}) / \Phi$ allowing the structural comparison between nanocomposites containing $18.0 \pm 0.3 \% \mathrm{v}$ of silica, coating agent octeo $(8 \% \mathrm{wt})$, and DPG, made with different polymer matrices $\mathrm{F}$ (squares), NF (circles), and a 50/50 mixture (diamonds). Arrows indicate the q-ranges of nanoparticle- and aggregate-scattering, respectively. Inset: Bound rubber results for the same samples.

A quantitative analysis confirming the impact of matrix composition has been performed following ref. ${ }^{41}$. It allows the determination of not only $\mathrm{R}_{\mathrm{agg}}$, but also the aggregate density (compacity $\kappa$ ), and thus the average aggregation number $\mathrm{N}_{\text {agg, }}$ all given in Table 1 . As in our previous work with a different polymer ${ }^{42}$, the more functionalized matrix chains are, the lower the aggregate radius determined through the Kratky representation, and the lower the $\kappa$. This tendency results in a decrease of the aggregation number by a factor of two. The evolution towards smaller aggregates is accompanied by the formation of a well-defined shoulder around $0.01 \AA^{-1}$ : nanocomposites formed with the end-functionalized polymer chains thus present the most prominent signature in $\mathrm{I}(\mathrm{q})$, indicative of the best dispersion of smallest possible aggregates. Moreover, the effect of the graftable chains can also be evidenced by bound rubber measurements, the results of which are also summarized in Table 1 and in the inset in Figure 1. Even with only un-functionalized polymer (NF), about half of the chains are not desorbed in a BR measurement, most probably due to weak crosslinking of the chains during mixing, or possibly chain adsorption on the filler. The high fraction of bound rubber with graftable chains indicates that grafting took place. We thus choose to study the impact of the small molecules, silane and DPG, only with fully functionalized, graftable matrices, by investigating how the dispersion evolves as the quantity of small molecules is reduced. 


\begin{tabular}{|c|c|c|c|c|c|c|}
\hline Sample & Coating & $\Phi_{\mathbf{S i}}(\boldsymbol{\%} \mathbf{v})$ & $\mathbf{R}_{\text {agg }}(\mathbf{n m})$ & $\boldsymbol{\kappa}(\boldsymbol{\%})$ & $\mathbf{N}_{\mathbf{a g g}}$ & \%BR \\
\hline $\mathrm{NF}$ & $\mathrm{C}_{8}$ & 18.3 & 33 & 70 & 67 & 46 \\
\hline $50 / 50$ & $\mathrm{C}_{8}$ & 18.2 & 33 & 51 & 47 & 57 \\
\hline $\mathrm{F}$ & $\mathrm{C}_{8}$ & 18.1 & 30 & 44 & 31 & 96 \\
\hline $\mathrm{F}$ & $\mathrm{C}_{12}$ & 18.1 & 30 & 45 & 31 & 98 \\
\hline $\mathrm{F}$ & $\mathrm{C}_{18}$ & 18.3 & 31 & 45 & 34 & 99 \\
\hline $\mathrm{F}$ & $4 \%$ wt $\mathrm{C}_{8}$ & 17.8 & 32 & 46 & 39 & 92 \\
\hline F & - & 17.5 & 34 & 47 & 48 & 88 \\
\hline F, no DPG & $\mathrm{C}_{8}$ & 18.1 & 36 & 50 & 61 & 88 \\
\hline F, no DPG & - & 17.4 & 38 & 48 & 67 & 69 \\
\hline
\end{tabular}

Table 1: Nanocomposite formulations with silica content $\Phi_{\mathrm{Si}}=18.0 \pm 0.3 \% \mathrm{v}$. Average radius of aggregates from Kratky analysis, compacity, aggregation number, and bound rubber of PNCs. All samples contain $8 \%$ wt $\mathrm{C}_{8}$ with respect to silica (resp., isomolar $\mathrm{C}_{12}, \mathrm{C}_{18}$ ) and $\mathrm{DPG}$, unless otherwise stated.

Impact of hydrophobic character of coating agent on nanocomposite structure: Subsequent to the choice of the polymer matrix, the coating agent needed to be chosen. We have varied the chemical nature of the coating molecule, via the length of its aliphatic chain. The macroscopic bound rubber values are almost identical $(96-99 \%)$ and are given in Table 1. This suggests that the chain length of the coating agent has only little influence on polymer grafting, which we have seen in Figure 1 to be an important driver of the PNC microstructure. The scattering results for an $18 \%$-nanocomposite, in a fully functionalized polymer matrix, with DPG and isomolar amounts of $\mathrm{C}_{8}$ (octeo, $8 \%$ wt with respect to silica), $\mathrm{C}_{12}$, or $\mathrm{C}_{18}$, are shown in Figure 2.

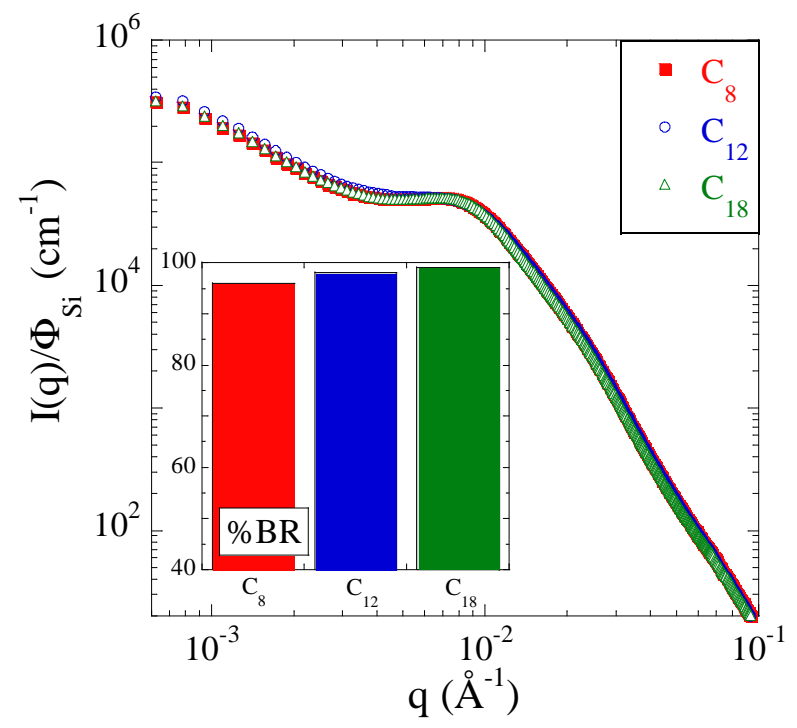

Figure 2: Reduced SAXS intensities allowing the structural comparison between nanocomposites containing $18.0 \pm 0.3 \% \mathrm{v}$ silica in a fully functionalized matrix with DPG, for three different isomolar silane coating agents: octeo ( $\mathrm{C}_{8}$, squares), $\mathrm{C}_{12}$ (circles), and $\mathrm{C}_{18}$ (triangles). The inset shows the bound rubber of the same PNCs.

Contrarily to what one might expect based on the formation of a more hydrophobic layer on the filler surface, potentially inducing a better compatibilization (and thus dispersion), there is no significant effect of the nature of the coating agent on the aggregate structure. Indeed, the three intensity curves 
superimpose almost perfectly. This surprising result may be due to a saturation effect, i.e., adding the same number of higher-mass agents would not increase the silica surface coverage. In this picture, $\mathrm{C}_{8}$ molecules already reach a perfect coverage, and $\mathrm{C}_{18}$ only modifies the thickness of the interfacial layer. Also, bound rubber measurements seem to show no impact on the efficiency of polymer grafting on the silica surface, further indicating similar surface coverage. In the rest of this article, the surface modification is thus performed with the $\mathrm{C}_{8}$ coating agent (octeo), which incidentally is also the industrially relevant one.

Impact of amount of coating agent on nanocomposite structure. In Figure 3a, the normalized small-angle intensities scattered by nanocomposites of various octeo contents used during the formulation, 0,4 , and $8 \%$ wt with respect to the filler, respectively, are shown, all other parameters being fixed, namely the filler content and DPG.
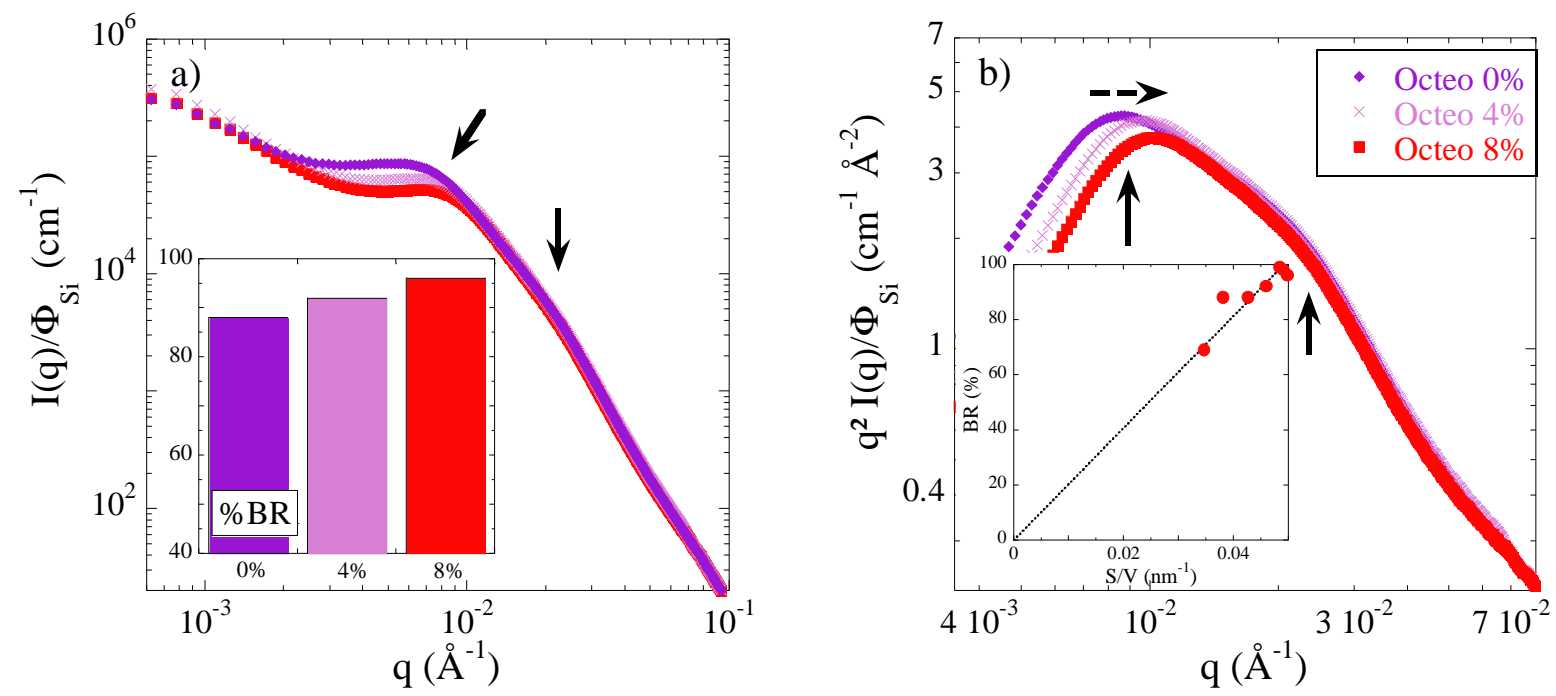

Figure 3: a) Reduced SAXS intensities allowing the structural comparison between nanocomposites containing $18.0 \pm 0.3 \% \mathrm{v}$ silica in a fully functionalized matrix with DPG, made with different amounts of coating agent octeo: 0 (diamonds), 4 (crosses), and $8 \%$ wt (squares). The inset shows the bound rubber of the same PNCs. Arrows indicate the q-ranges of particle- and aggregate-scattering, respectively. b) Kratky plot $\mathrm{q}^{2} \mathrm{I}(\mathrm{q})$ vs $\mathrm{q}$ highlighting the structural evolution (dashed arrow). Inset: All BR for functionalized polymers versus the specific surface of aggregates.

The scattered intensities in Figure 3a are characteristic of the same type of structure as discussed with Figure 1: obvious changes are induced by the presence of the coating agent, inducing better filler dispersion. A similar result has been reported in the literature for an ungrafted polymer matrix. ${ }^{19}$ In parallel, the fraction of bound rubber shown in the inset is seen to increase with the amount of octeo. Figure $3 \mathrm{a}$ is a key result of our study, as it demonstrates, to our knowledge for the first time, the delicate impact of the coating agent on structure in graftable matrices. The results of our quantitative analysis are given in Table 1. It reveals a decrease by more than $10 \%$ in the average aggregate radius $\mathrm{R}_{\mathrm{agg}}$, which is also seen in the Kratky representation in Figure $3 \mathrm{~b}$, with the shift of the maximum to 
higher q illustrated by the dashed arrow. This maximum is the direct consequence of the break in slope in I(q), indicative of the aggregate interaction, highlighted by the arrow around $0.01 \AA^{-1}$. From the analysis of the height of the signal in the 'aggregate region' up to $0.01 \AA^{-1}$, using the volume fraction of fractal branches determined independently from TEM pictures shown in Figure $4\left(\Phi_{\text {fract }} \approx 80 \%\right)$, the compacity is also found to decrease, as given in Table 1. As a result of the combination of decrease both in size and density, the average aggregation number $\mathrm{N}_{\text {agg }}$ is seen to diminish by $35 \%$, from 48 to 31. In the inset of Figure 3a, the bound rubber results are seen to slightly increase with increasing octeo content. As this coating agent does not contribute to any grafting of the polymer, this is a priori surprising. Our interpretation is that the decrease of $\mathrm{N}_{\mathrm{agg}}$ favors polymer-filler interactions, via a higher specific surface $\mathrm{S} / \mathrm{V}$ estimated by $3 * \Phi_{\mathrm{agg}} / \mathrm{R}_{\mathrm{agg}}$ in the inset of Figure $3 \mathrm{~b}$. Indeed, both bound rubber and $\mathrm{S} / \mathrm{V}$ increase by some $10 \%$. It cannot be decided, however, if the higher bound rubber is only adsorbed to the aggregates, possibly entangled with grafted chains making it difficult to desorb, or if the higher $\mathrm{S} / \mathrm{V}$ favors polymer access to surface and grafting. In parallel, the TEM images of the octeo-series in Figure 4 illustrate the progressive formation of smaller and smaller structures with increasing octeo content. Homogeneity on larger scales has been checked with lower resolution pictures. As expected, compatibilization of the originally hydrophilic filler surface with the hydrophobic polymer matrix through the coating agent induces the formation of smaller aggregates, i.e., a better - more "nano" - dispersion.

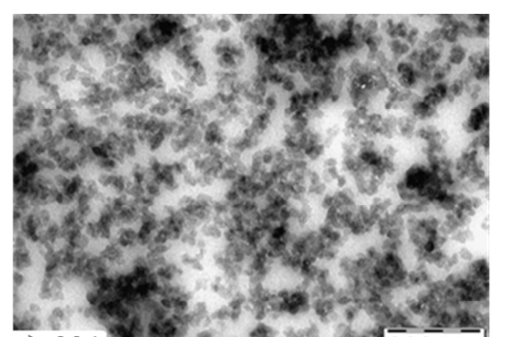

a) $0 \%$

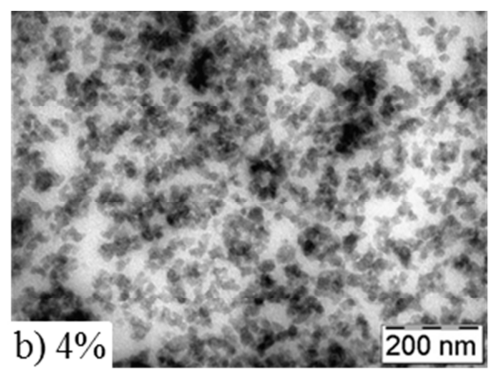

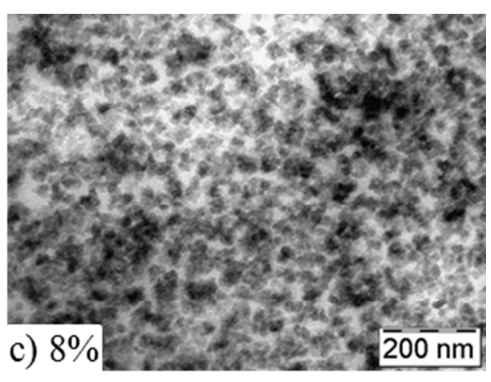

Figure 4: TEM images of nanocomposites containing $18.0 \pm 0.3 \% \mathrm{v}$ silica in a fully functionalized matrix with DPG, made with different amounts of coating agent octeo: 0,4 , and $8 \%$ wt with respect to silica.

Synergetic effect of coating agent and catalyzer on nanocomposite structure. The impact of the coating agent octeo being now characterized, the remaining question is the role of DPG. We have formulated and compared four different samples, containing either no agents, only octeo, only DPG, or both DPG and octeo. Concentrations have been set to the nominal values, i.e., 8\%wt octeo with respect to silica $(\approx 18 \% \mathrm{v}$ filler fraction), and $1 \%$ wt DPG with respect to polymer mass. The TEM images in Figures 4 and 5 seem to indicate that the characteristic size is larger in absence of both octeo and DPG, or only with octeo, than in presence of only DPG or both agents. For a quantitative analysis, however, the evolutions are too weak, and SAXS is needed. 

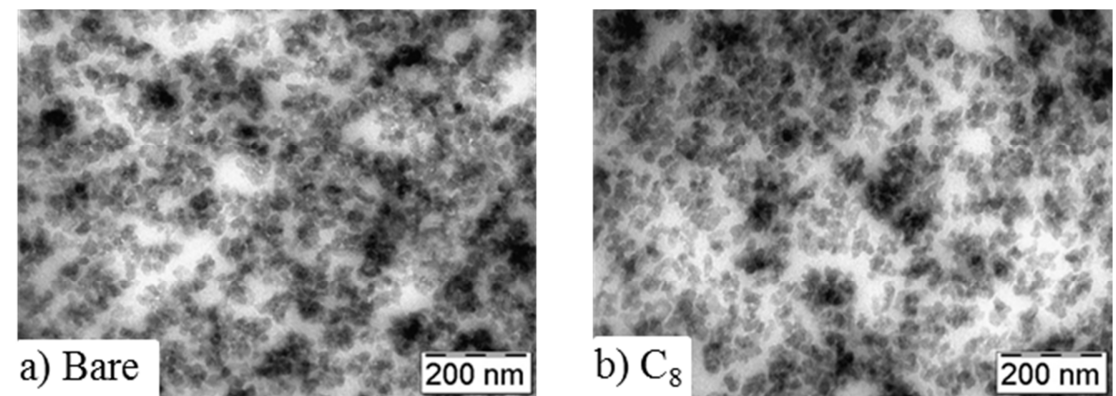

b) $\mathrm{C}_{8}$

$200 \mathrm{~nm}$

Figure 5: TEM images of nanocomposites containing $18.0 \pm 0.3 \% \mathrm{v}$ silica in a fully functionalized matrix: (a) without small molecules, (b) with octeo only ( $8 \%$ wt with respect to silica, no DPG). Images with only DPG, and with both octeo and DPG are presented in Figure 4.

In Figure 6a, the normalized scattered intensities are plotted for these samples, and the Kratky plots in Figure $6 \mathrm{~b}$ highlight the structural evolution.


Figure 6: a) Reduced SAXS intensities comparing the structure of nanocomposites containing $18.0 \pm 0.3 \% \mathrm{v}$ silica in a fully functionalized matrix: without small molecules (bare, triangles), with DPG only (diamonds), with octeo only (circles), and with both octeo and DPG (squares). The fraction of octeo (when present) is $8 \%$ wt. The inset shows the bound rubber of the same PNCs. b) Kratky representation of the same samples.

All the structures characterized by scattering in Figure 6a are again typical for this type of simplified industrial nanocomposites. The coating agent and DPG have a pronounced impact on the aggregate structure probed at intermediate $\mathrm{q}$, as one can see from the different intensity levels - keeping in mind the logarithmic scale: without any DPG or coating agent, the dispersion is worst, with the highest intensity in Figure 6a, while in presence of both - cf. the lowest, red curve in the figure -, the best dispersion is obtained. This is confirmed by the quantitative analysis of the Kratky plot (Figure 6b) presenting a shift to the right with added molecules, which leads to the average aggregate radius given 
in Table 1: $\mathrm{R}_{\text {agg }}$ decreases from 38 (no agents) to $30 \mathrm{~nm}$ (both agents). Simultaneously, the compacity and the aggregation number evolve from $\mathrm{\kappa}=48 \%$ to $44 \%$, and $\mathrm{N}_{\mathrm{agg}}$ from 67 to 31, respectively, while the bound rubber fraction increases from below $70 \%$ to above $95 \%$. The decrease in $\mathrm{N}_{\mathrm{agg}}$ is indicative of a better compatibility of the filler with the matrix, as there is more polymer in contact with fillers for smaller aggregates, and this leads to the highest bound rubber value in Figure 6a.

One might wonder if octeo and DPG produce a synergetic effect on the microstructure, or if only one of them might be effective. The two intermediate samples with either one of them have also been analyzed; cf. Figure 6 and Table 1. Adding only octeo decreases the aggregate radius to $36 \mathrm{~nm}$ and the aggregation number $\mathrm{N}_{\text {agg }}$ to 61 , whereas the bound rubber fraction increases. The latter behavior is consistent with the variation observed for different octeo contents in presence of DPG, cf. Figure 3, and probably has the same causes - a higher specific surface. Finally, adding only DPG has an unexpected effect on the structure, as it leads to a better dispersion than without any agent: the average aggregate radius is about $34 \mathrm{~nm}$, while compacity is $47 \%$ and the aggregation number $\mathrm{N}_{\text {agg }} \approx 48$. Such results may be due to the strong affinity of the basic DPG with the silica surface, which displays acidic character. DPG therefore preferentially adsorbs on the (untreated) silica, preventing by this way the formation of hydrogen bonds between hydroxyl groups of different nanoparticles. ${ }^{47,48}$ Note that the number density of silanol groups on silica has been shown to be correlated with the aggregate size in SBR nanocomposites. ${ }^{49}$ Comparison between the structural results with DPG only and octeo only further indicates that the efficiency of the coating agent alone is not optimal. The addition of DPG together with octeo is required to obtain the better dispersion (see Figure 6 and Table 1), DPG promoting the grafting reaction of the silane ${ }^{37}$. On the other hand, one may wonder if DPG also has a catalytic action on the grafting reaction of the polymer chains on the silica nanoparticles (all chains are expected to carry a Si-OH end-function). This does not seem to be the case as the BR values for DPG only and octeo only are identical. It follows that the $40 \%$-increase of the bound rubber fraction from no agents to both agents in Figure $6 \mathrm{a}$ is mostly driven by the structural evolution: the decrease of $\mathrm{N}_{\text {agg }}$ leads to a higher specific surface $(\approx+45 \%)$, favoring chain grafting. Confronting these findings with presence and absence of both molecules unambiguously proves that DPG and octeo are necessary to tune the large-scale filler structure efficiently.

In order to extend our discussion towards the influence of small molecules on crosslinked samples as they may be used in applications, we have first investigated the vulcanization kinetics given by the curing curves (torque versus time) of the pure matrix with and without DPG, see inset of Figure 7. As observed by Ramier et al. ${ }^{50}$, using only CBS (without DPG) shifts the beginning of the vulcanization process to longer times, which means that both accelerators are required for a rapid cure. In the meantime, we found that the presence of DPG impacts the final crosslinking density only by a few percent. We have then formulated four crosslinked samples in analogy to the four samples compared in Figure 6. The experimental difficulty with such samples is that the curing agents are added in the 
two-roll mill process, inducing slight anisotropy in the samples and thus intensities. Luckily, the average level of these intensities as shown in the next plot, however, varies strongly with the composition, making conclusions robust independently of anisotropy. In Figure 7, the same color code as for Figure 6 applies, comparing crosslinked samples with both DPG and octeo, with only one, or neither. The scattering of the corresponding uncrosslinked samples (Fig.6a) is superimposed in grey for convenience.

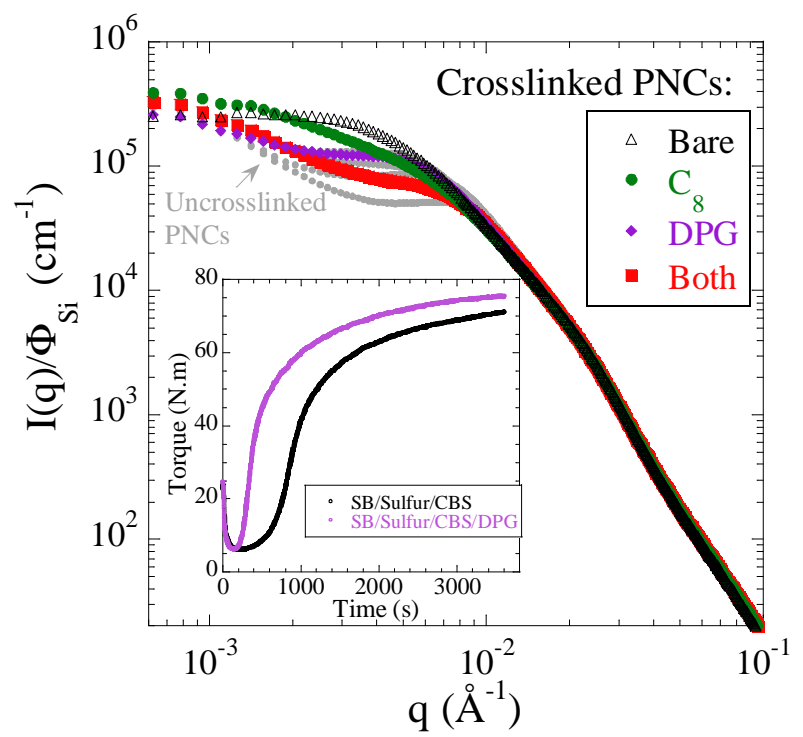

Figure 7: Reduced SAXS intensities comparing the structure of crosslinked nanocomposites containing $17.5 \pm 0.3 \% \mathrm{v}$ silica in a fully functionalized matrix: without small molecules (bare, triangles), with DPG only (diamonds), with octeo only (circles), and with both octeo and DPG (squares). The fraction of octeo (when present) is 8\%wt. The uncrosslinked curves shown in Figure 6 are superimposed in grey. Inset: Curing curves of unfilled polymer: $\mathrm{SB}+$ Sulfur+CBS, and $\mathrm{SB}+\mathrm{Sulfur}+\mathrm{CBS}+\mathrm{DPG}$.

In Figure 7, crosslinked and uncrosslinked structures are identical at high q, and at low q intensities tend to converge. In the intermediate q-range, all four intensity curves of crosslinked samples are higher by less than a factor of two than the uncrosslinked ones. This means crosslinking favors slightly the aggregation of silica nanoparticles, similarly to previous results ${ }^{51}$. For instance, comparing the intensity levels of the samples having both small molecules, the crosslinked one has bigger aggregates $\mathrm{R}_{\mathrm{agg}}(34 \mathrm{~nm}$, uncrosslinked: $32 \mathrm{~nm})$, about the same compacity ( $\mathrm{\kappa}=47 \%$, resp. $\left.44 \%\right)$, and a higher $\mathrm{N}_{\mathrm{agg}}$ $=46$ (resp. 31). It is unclear, however, if this increase in aggregation is due to the crosslinker which might influence interactions between particles, or if mechanical action during crosslinking has this effect. The most remarkable fact in Figure 7 is that the order of the curves is unchanged with respect to the uncrosslinked case. Our findings that both small molecules, DPG and octeo, are necessary to tune the large-scale filler structure efficiently thus remain valid also for crosslinked samples. 


\section{Conclusion}

We have investigated the impact of small molecules - coating agents and DPG added during formulation by solid-phase mixing - on the bound rubber fraction and on the microstructure of polymer nanocomposites made with disordered silica filler of industrial relevance. The bound rubber is found to be correlated with the microstructure (see inset of Figure 3b), suggesting a specific-surface effect, smaller aggregates offering more possibilities to bind polymer. The microstructure is characterized by small-angle scattering and TEM, with a particular focus on aggregates of primary NPs, which we have shown to be the fundamental structural units, cf. recent reviews ${ }^{44}, 52$. Accordingly, the structure can be quantitatively described with two independent measures, the average aggregate radius $\mathrm{R}_{\mathrm{agg}}$, and the average aggregation number $\mathrm{N}_{\mathrm{agg}}$, or equivalently, the compacity $\kappa$. Both have been shown to evolve with the coating agent content, higher concentrations of this additive giving better dispersion, i.e., smaller aggregates, presumably due to a better compatibility with the matrix. There was surprisingly no impact of the chain length of the coating agent on the nanocomposite microstructure, suggesting that short chains are sufficient to cover the nanoparticle surface. Concerning this latter point, one might nonetheless expect a modification of the local dynamics, caused by a plasticizing effect of the matrix by the longer aliphatic chains, a point which we hope to address in a future study. Along the same lines, one could also investigate if the absence of effect is due to saturation of the surface, e.g., by performing a study of longer molecules as a function of (lower) concentrations.

By confronting the dispersion of nanocomposites with and without the amine DPG, this molecule was unambiguously demonstrated to be necessary to promote the compatibilizing effect of the coating agent: there is a strong synergy between the amine and the coating agent. This conclusion was shown to remain true even for industrially more relevant crosslinked samples. By comparing bound rubber and structure to a sample containing only DPG, it was further shown that DPG does not catalyze the matrix end-grafting. Incidentally, this could be cross-checked in future studies with nanocomposites made only with a non graftable matrix, which was excluded here in order to obtain the strongest possible structural signature of synergy.

Acknowledgements: The authors are thankful for support by the ANR NANODYN project, grant ANR-14-CE22-0001-01 of the French Agence Nationale de la Recherche. We thank Benoit Moreaux (Solvay Silica) for helping with the preparation of the samples. TEM preparation at Solvay was done by Pauline Grau and Magalie Fontana. 


\section{References}

(1) Mark, J. E.; Erman, B.; Eirich, F. R., Science and Technology of Rubber. Academic Press: San Diego, 1994; p 367.

(2) Jancar, J.; Douglas, J. F.; Starr, F. W.; Kumar, S. K.; Cassagnau, P.; Lesser, A. J.; Sternstein, S. S.; Buehler, M. J. Current issues in research on structure-property relationships in polymer nanocomposites. Polymer 2010, 51 (15), 3321-3343.

(3) Schmidt, G.; Malwitz, M. M. Properties of polymer-nanoparticle composites. Current Opinion in Colloid \& Interface Science 2003, 8 (1), 103-108.

(4) Kumar, S. K.; Jouault, N.; Benicewicz, B.; Neely, T. Nanocomposites with Polymer Grafted Nanoparticles. Macromolecules 2013, 46 (9), 3199-3214.

(5) Jordan, J.; Jacob, K. I.; Tannenbaum, R.; Sharaf, M. A.; Jasiuk, I. Experimental trends in polymer nanocomposites - a review. Mater. Sci. Eng., A 2005, 393 (1-2), 1-11.

(6) Kim, W.-S.; Lee, D.-H.; Kim, I.-J.; Son, M.-J.; Kim, W.; Cho, S.-G. SBR/Organoclay Nanocomposites for the Application on Tire Tread Compounds. Macromol. Res. 2009, 17 (10), 776-784.

(7) Chevigny, C.; Jouault, N.; Dalmas, F.; Boue, F.; Jestin, J. Tuning the Mechanical Properties in Model Nanocomposites: Influence of the Polymer-Filler Interfacial Interactions. J. Polym. Sci., Part B: Polym. Phys. 2011, 49 (11), 781-791.

(8) Heinrich, G.; Dumler, H. B. Wet skid properties of filled rubbers and the rubber-glass transition. Rubber Chem. Technol. 1998, 71 (1), 53-61.

(9) Manoharan, P.; Naskar, K. Exploring a highly dispersible silica-elastomer composite for tire applications. J. Appl. Polym. Sci. 2016, 133 (24), 43531.

(10) Sarkawi, S. S.; Kaewsakul, W.; Sahakaro, K.; Dierkes, W. K.; Noordermeer, J. W. M. A Review on Reinforcement of Natural Rubber by Silica Fillers for Use in Low-Rolling Resistance Tyres. J. Rubber Res. 2015, 18 (4), 203-233.

(11) Wolff, S. Chemical Aspects of Rubber Reinforcement by Fillers. Rubber Chem. Technol. 1996, 69 (3), 325-346.

(12) Frohlich, J.; Niedermeier, W.; Luginsland, H. D. The effect of filler-filler and filler-elastomer interaction on rubber reinforcement. Compos Part A Appl Sci Manuf. 2005, 36 (4), 449-460.

(13) Hasse, A.; Klockmann, O.; Wehmeier, A.; Luginsland, H. D. Influence of the amount of diand polysulfane silanes on the crosslinking density of silica filled rubber compounds. Kautsch. Gummi, Kunstst. 2002, 55 (5), 236-243.

(14) Heinrich, G.; Vilgis, T. A. Why silica technology needs S-SBR in high performance tires? The physics of confined polymers in filled rubbers. Kautsch. Gummi, Kunstst. 2008, 61 (7-8), 368376. 
(15) Bachmann, J. H.; Sellers, J. W.; Wagner, M. P.; Wolf, R. F. Fine Particle Reinforcing Silicas and Silicates in Elastomers. Rubber Chem. Technol. 1959, 32 (5), 1286-1391.

(16) Wang, M.-J.; Wolff, S.; Donnet, J.-B. Filler-Elastomer Interactions. Part I: Silica Surface Energies and Interactions with Model Compounds. Rubber Chem. Technol. 1991, 64 (4), 559576.

(17) Wolff, S.; Wang, M.-J. Filler-Elastomer Interactions. Part IV. The Effect of the Surface Energies of Fillers on Elastomer Reinforcement. Rubber Chem. Technol. 1992, 65 (2), 329-342.

(18) Sternstein, S. S.; Zhu, A.-J. Reinforcement Mechanism of Nanofilled Polymer Melts As Elucidated by Nonlinear Viscoelastic Behavior. Macromolecules 2002, 35 (19), 7262-7273.

(19) Bouty, A.; Petitjean, L.; Degrandcourt, C.; Gummel, J.; Kwaśniewski, P.; Meneau, F.; Boué, F.; Couty, M.; Jestin, J. Nanofiller Structure and Reinforcement in Model Silica/Rubber Composites: A Quantitative Correlation Driven by Interfacial Agents. Macromolecules 2014, 47 (15), 5365-5378.

(20) Yan, H.; Tian, G.; Sun, K.; Zhang, Y.; Zhang, Y. Effect of silane coupling agent on the polymer-filler interaction and mechanical properties of silica-filled NR. J. Polym. Sci., Part B: Polym. Phys. 2005, 43 (5), 573-584.

(21) Wu, Y.-P.; Zhao, Q.-S.; Zhao, S.-H.; Zhang, L.-Q. The influence of in situ modification of silica on filler network and dynamic mechanical properties of silica-filled solution styrene-butadiene rubber. J. Appl. Polym. Sci. 2008, 108 (1), 112-118.

(22) Schmitt Pauly, C.; Genix, A.-C.; Alauzun, J. G.; Sztucki, M.; Oberdisse, J.; Mutin, P. H. Surface modification of alumina-coated silica nanoparticles in aqueous sols with phosphonic acids and impact on nanoparticle interactions. Phys. Chem. Chem. Phys. 2015, 17 (29), 1917319182.

(23) Schmitt Pauly, C.; Genix, A.-C.; Alauzun, J. G.; Jestin, J.; Sztucki, M.; Mutin, P. H.; Oberdisse, J. Structure of alumina-silica nanoparticles grafted with alkylphosphonic acids in poly(ethylacrylate) nanocomposites. Polymer 2016, 97, 138-146.

(24) Ramier, J.; Gauthier, C.; Chazeau, L.; Stelandre, L.; Guy, L. Payne effect in silica-filled styrene-butadiene rubber: Influence of surface treatment. J. Polym. Sci., Part B: Polym. Phys. 2007, 45 (3), 286-298.

(25) Stockelhuber, K. W.; Svistkov, A. S.; Pelevin, A. G.; Heinrich, G. Impact of Filler Surface Modification on Large Scale Mechanics of Styrene Butadiene/Silica Rubber Composites. Macromolecules 2011, 44 (11), 4366-4381.

(26) Yatsuyanagi, F.; Suzuki, N.; Ito, M.; Kaidou, H. Effects of surface chemistry of silica particles on the mechanical properties of silica filled styrene-butadiene rubber systems. Polymer Journal 2002, 34 (5), 332-339.

(27) Suzuki, N.; Ito, M.; Yatsuyanagi, F. Effects of rubber/filler interactions on deformation behavior of silica filled SBR systems. Polymer 2005, 46 (1), 193-201. 
(28) Gauthier, C.; Reynaud, E.; Vassoille, R.; Ladouce-Stelandre, L. Analysis of the non-linear viscoelastic behaviour of silica filled styrene butadiene rubber. Polymer 2004, 45 (8), 27612771.

(29) Luginsland, H. D.; Frohlich, J.; Wehmeier, A. Influence of different silanes on the reinforcement of silica-filled rubber compounds. Rubber Chem. Technol. 2002, 75 (4), 563-579.

(30) Mele, P.; Marceau, S.; Brown, D.; de Puydt, Y.; Alberola, N. D. Reinforcement effects in fractal-structure-filled rubber. Polymer 2002, 43 (20), 5577-5586.

(31) Payne, A. R. The dynamic properties of carbon black-loaded natural rubber vulcanizates. Part I. J. Appl. Polym. Sci. 1962, 6 (19), 57-63.

(32) Pérez-Aparicio, R.; Vieyres, A.; Albouy, P.-A.; Sanséau, O.; Vanel, L.; Long, D. R.; Sotta, P. Reinforcement in Natural Rubber Elastomer Nanocomposites: Breakdown of Entropic Elasticity. Macromolecules 2013, 46 (22), 8964-8972.

(33) Conzatti, L.; Costa, G.; Castellano, M.; Turturro, A.; Negroni, F. M.; Gerard, J. F. Morphology and viscoelastic behaviour of a silica filled styrene/butadiene random copolymer. Macromol. Mater. Eng. 2008, 293 (3), 178-187.

(34) Castellano, M.; Conzatti, L.; Turturro, A.; Costa, G.; Busca, G. Influence of the silane modifiers on the surface thermodynamic characteristics and dispersion of the silica into elastomer compounds. J. Phys. Chem. B 2007, 111 (17), 4495-4502.

(35) Mujtaba, A.; Keller, M.; Ilisch, S.; Radusch, H. J.; Thurn-Albrecht, T.; Saalwachter, K.; Beiner, M. Mechanical Properties and Cross-Link Density of Styrene-Butadiene Model Composites Containing Fillers with Bimodal Particle Size Distribution. Macromolecules 2012, 45 (16), 6504-6515.

(36) Schaal, S.; Coran, A. Y.; Mowdood, S. K. The Effects of Certain Recipe Ingredients and Mixing Sequence on the Rheology and Processability of Silica- and Carbon Black-Filled Tire Compounds. Rubber Chem. Technol. 2000, 73 (2), 240-252.

(37) Penot, C., Rubber composition for a tire comprising a reinforcing inorganic filler and an (inorganic filler/elastomer) coupling system. In Google Patents: 2005.

(38) Kaewsakul, W.; Sahakaro, K.; Dierkes, W. K.; Noordermeer, J. W. M. Optimization of mixing conditions for silica-reinforced natural rubber tire tread compounds. Rubber Chem. Technol. 2012, 85 (2), 277-294.

(39) Choi, S.-S. Difference in bound rubber formation of silica and carbon black with styrenebutadiene rubber. Polymer. Adv. Tech. 2002, 13 (6), 466-474.

(40) Castellano, M.; Conzatti, L.; Costa, G.; Falqui, L.; Turturro, A.; Valenti, B.; Negroni, F. Surface modification of silica: 1. Thermodynamic aspects and effect on elastomer reinforcement. Polymer 2005, 46 (3), 695-703. 
(41) Baeza, G. P.; Genix, A. C.; Degrandcourt, C.; Petitjean, L.; Gummel, J.; Couty, M.; Oberdisse, J. Multiscale Filler Structure in Simplified Industrial Nanocomposite Silica/SBR Systems Studied by SAXS and TEM. Macromolecules 2013, 46 (1), 317-329.

(42) Baeza, G. P.; Genix, A. C.; Degrandcourt, C.; Petitjean, L.; Gummel, J.; Schweins, R.; Couty, M.; Oberdisse, J. Effect of Grafting on Rheology and Structure of a Simplified Industrial Nanocomposite Silica/SBR. Macromolecules 2013, 46 (16), 6388-6394.

(43) Baeza, G. P.; Genix, A. C.; Degrandcourt, C.; Gummel, J.; Mujtaba, A.; Saalwächter, K.; Thurn-Albrecht, T.; Couty, M.; Oberdisse, J. Studying twin-samples provides evidence for a unique structure-determining parameter in simplified industrial nanocomposites. ACS Macro Letters 2014, 3, 448-452.

(44) Genix, A. C.; Baeza, G. P.; Oberdisse, J. Recent advances in structural and dynamical properties of simplified industrial nanocomposites. Eur. Polym. J. 2016, 85, 605-619.

(45) Reuvekamp, L. A. E. M.; Brinke, J. W. t.; Swaaij, P. J. v.; Noordermeer, J. W. M. Effects of Time and Temperature on the Reaction of Tespt Silane Coupling Agent During Mixing with Silica Filler and Tire Rubber. Rubber Chem. Technol. 2002, 75 (2), 187-198.

(46) Baeza, G. P.; Genix, A. C.; Degrandcourt, C.; Gummel, J.; Couty, M.; Oberdisse, J. Mechanism of aggregate formation in simplified industrial silica styrene-butadiene nanocomposites: effect of chain mass and grafting on rheology and structure. Soft Matter 2014, 10, 6686-6695.

(47) Zaborski, M.; Donnet, J. B. Activity of fillers in elastomer networks of different structure. Macromol. Symp. 2003, 194 (1), 87-100.

(48) Kosmalska, A.; Zaborski, M.; Ślusarski, L. Adsorption of curatives and activity of silica toward elastomers. Macromol. Symp. 2003, 194 (1), 269-276.

(49) Yatsuyanagi, F.; Suzuki, N.; Ito, M.; Kaidou, H. Effects of secondary structure of fillers on the mechanical properties of silica filled rubber systems. Polymer 2001, 42 (23), 9523-9529.

(50) Ramier, J.; Chazeau, L.; Gauthier, C.; Guy, L.; Bouchereau, M. N. Influence of Silica and its Different Surface Treatments on the Vulcanization Process of Silica Filled SBR. Rubber Chem. Technol. 2007, 80 (1), 183-193.

(51) Baeza, G. P.; Genix, A.-C.; Paupy-Peyronnet, N.; Degrandcourt, C.; Couty, M.; Oberdisse, J. Revealing nanocomposite filler structures by swelling and small-angle X-ray scattering. Farad. Discuss. 2016, 186, 295-309

(52) Genix, A.-C.; Oberdisse, J. Structure and dynamics of polymer nanocomposites studied by Xray and neutron scattering techniques. Current Opinion in Colloid \& Interface Science 2015, 20 (4), 293-303. 


\section{FOR TABLE OF CONTENTS USE ONLY}

Synergistic effect of small molecules on large-scale structure of simplified industrial nanocomposites

Dafne Musino, Anne-Caroline Genix, Caroline Fayolle, Aurélie Papon, Laurent Guy, Natalia Meissner, Radosław Kozak, Pawel Weda, Thomas Bizien, Thomas Chaussée, Julian Oberdisse
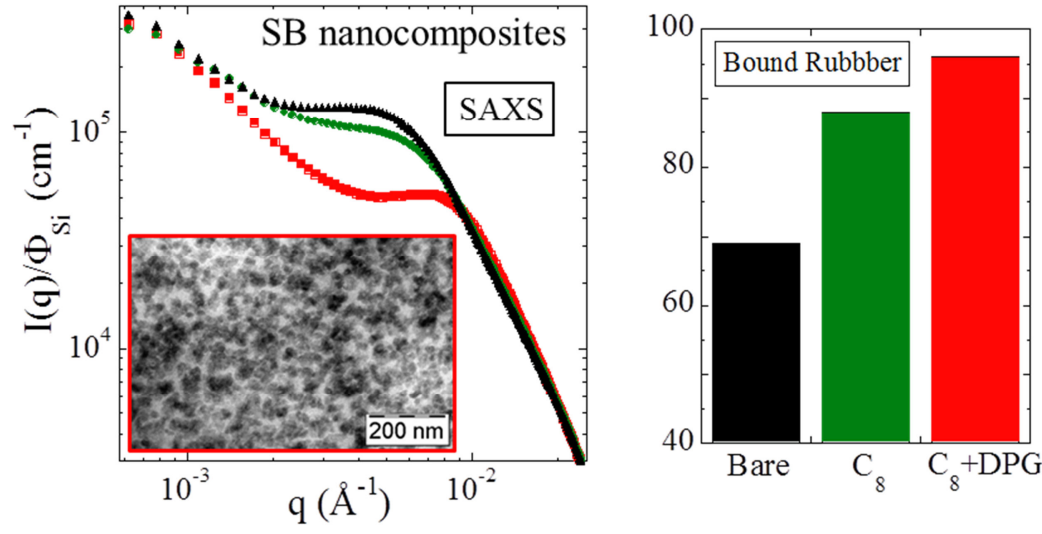